\title{
Scientia Agropecuaria
}

\section{Efecto de la altura de poda de formación en la arquitectura de plantas de camu camu (Myrciaria dubia H.B.K. Mc Vaugh) en la estación experimental del IIAP, Ucayali, Perú.}

\section{Effect of pruning height on the architecture of plants of camu camu (Myrciaria dubia HBK Mc Vaugh) in the experimental station of IIAP, Ucayali, Peru.}

\author{
Carlos Abanto ${ }^{1, *}$, José Sánchez Choy ${ }^{1}$, Wilson Saldaña ${ }^{1}$, Marden Paifa ${ }^{1}$, Edvan Alves Chagas ${ }^{2}$ \\ ${ }^{1}$ Instituto de Investigaciones de la Amazonia Peruana-IIAP PROBOSQUES-PIBA.CFB Km 12,400. Pucallpa (Perú). \\ ${ }^{2}$ Empresa Brasilera de Pesquisas Agropecuárias - EMBRAPA Roraima-Rodovia BR-174, Km 8 - Distrito Industrial-Brasil.
}

Recibido 05 junio 2011; aceptado 30 junio 2011

\begin{abstract}
Resumen
Con el objetivo de evaluar la respuesta de las plantas de camu camu en plantaciones iniciales sometidas a podas de formación, se instaló un experimento en la EE - IIAP-Ucayali, bajo un Diseño de Bloques Completos al Azar con 3 repeticiones, considerando 20 plantas por unidad experimental. Los tratamientos consistieron en realizar podas a diferentes alturas desde la base del tallo, se consideró T0 [testigo sin poda]; T1 [poda a $10 \mathrm{~cm}$ de la base]; T2 [poda a $20 \mathrm{~cm}$ de la base] y T3 [poda a $40 \mathrm{~cm}$ de la base] en plantas de $1.5 \mathrm{~m}$ de altura en promedio, procedentes de semillas de la cuenca del rio Putumayo. Se evaluó el número de brotes, longitud de brotes, altura de planta, diámetro basal, diámetro de copa y número de ramas. Luego de 15 meses de evaluación se encontró diferencias significativas entre las variables estudiadas excepto en diámetro de copa. En altura sobresale el T0 [testigo sin poda]; los tratamientos restantes se comportaron de forma similar, superando al testigo en $267 \%$. Así mismo, para el diámetro basal se halló que el T1 [poda a $10 \mathrm{~cm}$ de la base] se comportó mejor con un valor promedio de $1.96 \mathrm{~cm}$ frente al testigo con $1.7 \mathrm{~cm}$; Para el número de ramas la poda tuvo una influencia positiva, con un promedio de 13.4 ramas frente al T0 [testigo sin poda]; con 3.1 ramas, con lo cual se demostró que la poda de formación incrementa hasta un $432.3 \%$.
\end{abstract}

Palabras clave: Brotes, copa, ramas, diámetro basal, altura de planta.

\begin{abstract}
In order to evaluate the response camu camu's plants in plantations initial undergoing training pruning, an experiment was installed in EE - IIAP-Ucayali, under a design randomized complete block, with 3 replications, making use of 20 plants per experimental unit. The pruning treatments were performing at different heights from the base of the stem, it was considered T0 [witness without pruning], T1 [pruning to 10 $\mathrm{cm}$ from the base], T2 [pruning to $20 \mathrm{~cm}$ from the base] and T3 [pruning to $40 \mathrm{~cm}$ from the base] in plants of known provenance. The investigation was directed to evaluate the number of shoots, shoot growth, plant height, basal diameter, crown diameter and number of branches. After of 9 months of evaluation was found significant differences between the variables except in top diameter. In the variable Height, treatment T0 [witness without pruning] outstanding because they were not pruned, among the remaining treatments has similarly behaved, surpassing the control treatment by $267 \%$. In addition to this, the basal diameter was found that $\mathrm{T} 1$ [pruning to $10 \mathrm{~cm}$ from the base] has a better performed with an average value of $1.96 \mathrm{~cm}$ compared with the control $(1.7 \mathrm{~cm})$. The pruning had a positive influence in the number of branches, with an average of 13.4 branches compared with T0 that get 3.1 branches on average, its shows that the pruning can increased up the production of branches to $432.3 \%$.
\end{abstract}

Keywords: shoots, glass, branches, basal diameter, plant height.

\section{Introducción}

El Camu Camu arbustivo es un importante antioxidante natural que estimula e incrementa la capacidad de defensa del organismo debido a su alta concentración de Ácido Ascórbico: 2700 a 3200 mg

\footnotetext{
* Autor para correspondencia

Email: carforestal24@gmail.com (C. Abanto)
} 
AA/100 g de pulpa (IIAP, 2001), 100 veces más Vitamina $C$ que la naranja; además contiene: Vitamina $\mathrm{A}, \mathrm{B}$, fósforo, proteínas, hierro, calcio Beta-caroteno, Niacina, Riblofavina, Tiamina, niacina y es rico en bioflavonoides (PROMPEX, 1998). Debido a la importancia del cultivo, existe la necesidad de promover bajo el enfoque comercial, la cual involucra insertar nuevas tecnologías de manejo agronómico.

Los primeros modelos tecnológicos desarrollados por las instituciones de investigación, estuvieron orientados al establecimiento del cultivo en parcelas pequeñas de productores ubicados en las riberas de los ríos amazónicos (Abanto, 2011). El comportamiento de las plantas de camu camu en estado natural muestra la exigencia de podas de formación; debido al crecimiento elongado de dos o tres ramas que alcanzan hasta 3 metros en los primeros años y con tendencia a doblarse cuando inicia la producción; en muchos casos rompiendo las ramas por su inestabilidad debido a su longitud y peso del fruto (Abanto, 2010). En ese sentido para lograr el manejo eficiente del cultivo, es necesario implementar una serie de técnicas y estrategias de manejo, la cual va traducirse en incremento de la producción y en plantaciones más rentables. Una de estas técnicas de manejo es la poda de formación en plantaciones iniciales la cual busca conseguir un tamaño adecuado de planta para facilitar la cosecha y armar una estructura de manera que las ramas queden bien iluminadas para favorecer la producción de frutas en cantidad y calidad; del mismo modo Avilán et al. (2000) afirma que la poda de formación se efectúa en los primeros estadios de la planta, para proporcionarle una estructura adecuada de ramas, es decir, de inserción equidistante entre sí, para que los ramos y la cosecha se distribuyan mejor, evitando el desgarramiento de las ramas por el peso de los frutos. Una de las podas de formación más comúnmente utilizadas es la poda en forma de vaso que se puede realizar en la mayoría de las especies. Esta poda consiste en rebajar el eje central de la planta a unos 60-80 cm. de altura y luego elegir de tres a cuatro ramas bien distribuidas separadas unas de otras de 15 a $20 \mathrm{~cm}$. y con direcciones diferentes (Morris y Suarez, s.f.).

La presente investigación tuvo como objetivo verificar la respuesta de la poda de formación sobre la emisión del número y longitud de brotes, de la misma forma en el diámetro basal, altura de la planta y número de ramas.

\section{Materiales y métodos}

El experimento fue realizado en la EEIIAP-Ucayali, jurisdicción del distrito de Yarinacocha, provincia de Coronel Portillo, Región Ucayali, ubicado en el Km. 12.4 de la Carretera Federico Basadre en la ciudad de Pucallpa ( $8^{\circ} 22^{\prime} 31^{\prime}$ ' de latitud Sur y $74^{\circ} 34^{\prime} 35^{\prime}$ ' de longitud Oeste), a una altitud de $154 \mathrm{msnm}$; la precipitación y temperatura promedio registrada en el año 2009 fue de 1047.16 $\mathrm{mm}$ y de $26.15^{\circ} \mathrm{C}$, en el año 2010 fue de $800.38 \mathrm{~mm}$ y una temperatura promedio de $26.85^{\circ} \mathrm{C}$. Se instaló en marzo del 2009 en un área de $1024 \mathrm{~m}^{2}$ con 240 plantas sembradas a un distanciamiento de $2 \times 2$ $\mathrm{m}$, a una edad de 1.5 años y con una altura promedio de $1.5 \mathrm{~m}$; el suelo es un ultisol (suelo de altura), de textura franco arcilloso, deficiente materia orgánica $(1.36 \%)$, fósforo (2.28 ppm) y potasio (0.15 CMol/litro) con un $\mathrm{pH}$ de 5.78, saturación de aluminio de $38.26 \%$ y un CIC de $4.18 \mathrm{CMol} /$ litro. Se utilizó un diseño de bloques completos al azar (DBCA) y 3 repeticiones. Se aplicaron 4 tratamientos, un testigo T0 [plantas sin poda] y 03 con poda a diferentes alturas en el mismo día: T1 [poda a $10 \mathrm{~cm}$ de la base]; T2 [poda a $20 \mathrm{~cm}$ de la base] y T3 [poda a $40 \mathrm{~cm}$ de la base]. Antes de aplicar la primera poda del tallo se tomaron datos de altura $(\mathrm{cm})$ y diámetro basal $(\mathrm{cm})$ para determinar si el material a intervenir tenia 
similares características; permitiendo saber si los resultados son atribuidos a los tratamientos. La evaluación de número de brotes se realizó dos veces: a los 15 y 90 días. El crecimiento de brotes se realizó cuatro veces: 15, 30, 60 y 90 días (Octubre - Diciembre del 2009). Posteriormente se realizó un manejo de poda de formación siguiendo las sugerencias de Barrantes (2006), quien afirma que la primera poda de formación en guayaba se debe realizar de los 20 a $50 \mathrm{~cm}$ en el tronco principal. Luego se seleccionaron 3 ó 4 ramas (ramas madre). Estas ramas deben estar separadas a un ángulo de unos $45^{\circ}$. Cuando el color café de la rama alcanzó unos $40 \mathrm{~cm}$ de largo fue el momento de podar nuevamente, con el fin de que ésta pueda sostener la fruta y la rama no se deforme. También se siguieron las indicaciones de López y Casanova (2006). Se realizó un raleo de brotes, dejando los más vigorosos, grandes y lignificados con una orientación equidistante para que conformen los nuevos brazos (ramas) de la arquitectura de la planta; seguidamente estas y las subsiguientes fueron podadas a $30 \mathrm{~cm}$ cada tres meses escalonadamente durante un año. Al mismo tiempo se evaluó la altura de planta $(\mathrm{cm})$, diámetro basal $(\mathrm{cm})$, diámetro de copa $(\mathrm{cm})$ y número de ramas. Los resultados del ANVA y la prueba estadística de Tukey $(\alpha=0.05 \%)$ fueron analizados utilizando el programa estadístico SPSS (Versión 17).

\section{Resultados y discusión}

En la evaluación inicial de altura (m) y diámetro basal $(\mathrm{cm})$ (Figura 1) de las plantas de camu camu se observó que existe una distribución similar, con valores que van desde 1.49 y $1.54 \mathrm{~m}$ para altura y 0.84 hasta $0.90 \mathrm{~cm}$ para diámetro basal. Se encontró que ambas variables se comportaron estadísticamente iguales (a un nivel de confianza superior al 95\%) (Tabla 1). Esto es un buen resultado base porque cualquier diferencia que se pueda lograr después de la poda será en gran parte atribuida a los efectos del tratamiento.

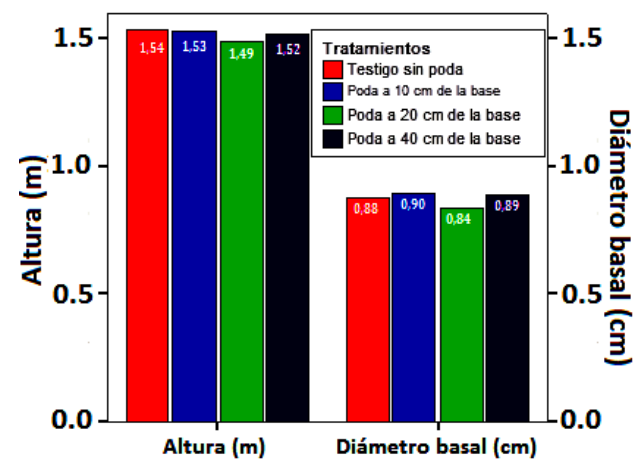

Figura 1. Altura $(\mathrm{m})$ y diámetro basal $(\mathrm{cm})$ inicial promedio de las plantas de camu camu antes de iniciar los tratamientos de podas de formación.

\section{Tabla 1}

Análisis de Variancia para altura (m) y Diámetro Basal $(\mathrm{cm})$ de plantas de camu camu antes de aplicar los tratamientos de podas de formación.

\begin{tabular}{ccccc}
\hline Variable & Fuente & GL & F & Significación \\
\hline \multirow{3}{*}{ Altura de } & Rep. & 2 & 4.357 & 0.014 \\
plantas (cm) & Tmto. & 3 & 0.542 & 0.654 \\
& Error & 234 & & \\
& Total & 239 & & \\
\hline \multirow{3}{*}{ Diámetro } & Rep. & 2 & 1.925 & 0.148 \\
basal $(\mathrm{cm})$ & Tmto. & 3 & 1.408 & 0.241 \\
& Error & 234 & & \\
& Total & 239 & & \\
\hline
\end{tabular}

En el análisis de variancia para el número de brotes; evaluados a los 15 y 90 días después de la primera poda, se observa que existen diferencias significativas entre tratamientos a un nivel de confianza superiores al 95\% (Tabla 2).

\section{Tabla 2}

Análisis de Variancia para $\mathrm{N}^{\circ}$ de brotes a los 15 y 90 días después de haber aplicado los tratamientos.

\begin{tabular}{lcccc}
\hline Variable & Fuente & GL & $\mathrm{F}$ & Significación \\
\hline \multirow{2}{*}{$\mathrm{N}^{\circ}$ de brotes } & Rep. & 2 & 4.357 & 0.431 \\
a los 15 días & Tmto. & 2 & 0.542 & 0.028 \\
& Error & 71 & & \\
& Total & 75 & & \\
\hline \multirow{2}{*}{$\mathrm{N}^{\circ}$ de brotes } & Rep. & 2 & 1.925 & 0.061 \\
a los 90 días & Erro. & 3 & 1.408 & 0.000 \\
& Total & 224 & & \\
\hline
\end{tabular}


En la Tabla 3 se observa que el mayor número de brotes lo obtuvieron los tratamientos T1, T3 у T2 con 6, 6 у 7 brotes respectivamente, no mostrando diferencias significativas entre podas; siendo significativamente superiores que el Testigo que sólo tuvo un promedio de 3 brotes; esto corrobora lo dicho por Zeledon y Wan (1994), quienes afirmaron que cuando se realiza el despunte de una planta que tiene entre 40 a $60 \mathrm{~cm}$ de alto, se provoca el desarrollo de brotes para posteriormente realizar la poda de formación.

\section{Tabla 3}

Prueba de Tukey para el número de brotes a los 15 días después de haber aplicado los tratamientos.

\begin{tabular}{lccc}
\hline \multirow{2}{*}{ DHS de Tukey ${ }^{\mathrm{a}, \mathrm{b}, \mathrm{c}}$} & & \multicolumn{2}{c}{$\begin{array}{c}\text { Significación } \\
(\alpha=0.05)\end{array}$} \\
\cline { 2 - 4 } & & \multicolumn{2}{c}{ Subconjunto } \\
\hline Tratamiento & Promedio & 1 & 2 \\
\hline T0 [Testigo sin poda] & 3 & $\mathrm{a}$ & \\
T3 [Poda a $40 \mathrm{~cm}$ ] & 6 & & $\mathrm{~b}$ \\
T1 [Poda a $10 \mathrm{~cm}$ ] & 6 & & $\mathrm{~b}$ \\
T2 [Poda a $20 \mathrm{~cm}$ ] & 7 & & $\mathrm{~b}$ \\
\hline
\end{tabular}

En la Tabla 4 se observa que el mayor número de brotes a los 90 días lo obtuvo el Testigo con un valor promedio de 10.67 unidades; siendo significativamente superior que T4 y $\mathrm{T} 1$ con 7.42 y 6.25 brotes, respectivamente.

\section{Tabla 4}

Prueba de Tukey para el $\mathrm{N}^{\mathrm{o}}$ de brotes a los 90 días después de haber aplicado los tratamientos.

\begin{tabular}{lcccc}
\hline & & \multicolumn{3}{c}{$\begin{array}{c}\text { Significación } \\
(\alpha=0.05)\end{array}$} \\
\cline { 2 - 5 } DHS de Tukey ${ }^{\mathrm{a}, \mathrm{b}, \mathrm{c}}$ & & \multicolumn{4}{c}{ Subconjunto } \\
\hline Tratamiento & Promedio & 1 & 2 & 3 \\
\hline T3 [Poda a 40 cm] & 6.25 & $\mathrm{a}$ & & \\
T1 [Poda a 10 cm] & 7.42 & $\mathrm{a}$ & $\mathrm{b}$ & \\
T2 [Poda a 20 cm] & 8.77 & & $\mathrm{~b}$ & $\mathrm{c}$ \\
T0 [Testigo sin poda] & 10.67 & & & $\mathrm{c}$ \\
\hline
\end{tabular}

En el análisis de variancia (Tabla 5) para la variable longitud de brotes a los 15 y 90 días se observa que existen diferencias significativas entre tratamientos a un nivel de confianza superiores al $95 \%$.

\section{Tabla 5}

Análisis de Variancia para Longitud de brotes (cm) a los 15 y 90 días después de haber aplicado los tratamientos.

\begin{tabular}{ccccc}
\hline Variable & Fuente & GL & F & Significación \\
\hline Longitud & Rep. & 2 & 7.59 & 0.00 \\
(cm) de & Tmto. & 3 & 41.64 & 0.00 \\
brotes a los & Error & 153 & & \\
15 días & Total & 158 & & \\
\hline Longitud & Rep. & 2 & 23.48 & 0.00 \\
(cm) de & Tmto. & 3 & 191.87 & 0.00 \\
brotes a los & Error & 221 & & \\
90 días & Total & 226 & & \\
\hline
\end{tabular}

En la tabla 6 se observa que la mayor longitud de brotes a los 15 días lo obtuvo el T0 con $4.56 \mathrm{~cm}$, siendo significativamente superior que los tratamientos $\mathrm{T} 1, \mathrm{~T} 2$ y $\mathrm{T} 3$ que solo alcanzaron un valor promedio de 0.33 ; 0.64 y $0.99 \mathrm{~cm}$ respectivamente.

\section{Tabla 6}

Prueba de Tukey para longitud de brotes a los 15 días después de haber aplicado los tratamientos.

\begin{tabular}{lccc}
\hline DHS de Tukey & & \multicolumn{2}{c}{$\begin{array}{c}\text { Significación } \\
(\alpha=0.05)\end{array}$} \\
\cline { 2 - 4 } & & \multicolumn{2}{c}{ Subconjunto } \\
\hline Tratamiento & Promedio & 1 & 2 \\
\hline T1 [Poda a $10 \mathrm{~cm}$ ] & 0.33 & $\mathrm{a}$ & \\
T2 [Poda a $20 \mathrm{~cm}$ ] & 0.64 & $\mathrm{a}$ & \\
T3 [Poda a $40 \mathrm{~cm}$ ] & 0.99 & $\mathrm{a}$ & b \\
T0 [Testigo sin poda] & 4.56 & & b \\
\hline
\end{tabular}

En la Tabla 7 se observa que la mayor longitud de brotes a los 90 días lo obtuvo el T2 [Poda a $20 \mathrm{~cm}$ ] con $54.73 \mathrm{~cm}$, siendo significativamente superior que los tratamientos T0, T3 y $\mathrm{T} 1$ que solo alcanzaron un valor promedio de 6.3; 44.2 y $47.38 \mathrm{~cm}$ respectivamente, indicando que existe mayor crecimiento de brotes cuando las plantas son podadas a 20 $\mathrm{cm}$ de altura desde la base del tallo. 
Tabla 7

Prueba de Tukey para longitud de brotes a los 90 días después de haber aplicado los tratamientos.

\begin{tabular}{lcccc}
\hline \multirow{2}{*}{ DHS de Tukey a,b,c } & & \multicolumn{3}{c}{$\begin{array}{c}\text { Significación } \\
(\alpha=0.05)\end{array}$} \\
\cline { 2 - 5 } & & \multicolumn{3}{c}{ Subconjunto } \\
\hline Tratamiento & Promedio & 1 & 2 & 3 \\
\hline T0 [Testigo sin poda] & 6.3 & $\mathrm{a}$ & & \\
T3 [Poda a $40 \mathrm{~cm}$ ] & 44.2 & & $\mathrm{~b}$ & \\
T1 [Poda a $10 \mathrm{~cm}$ ] & 47.38 & & $\mathrm{~b}$ & \\
T2 [Poda a $20 \mathrm{~cm}$ ] & 54.73 & & & $\mathrm{c}$ \\
\hline
\end{tabular}

El comportamiento de los brotes en el tratamiento Testigo sin poda, es lo que se esperaba por que el brotamiento ocurre en cualquier parte del tallo y según reportado en la evaluación inicial contábamos con plantas superior a $1.5 \mathrm{~m}$ de altura; la cual dio espacio suficiente para incrementar el número de brotes; dando como resultado brotes de diferentes tamaños y expresiones de vigor, por lo que no indicaron que todos sean eficientes y conformen la arquitectura ideal.

La evaluación de longitud de brotes $(\mathrm{cm})$ desde los 15 hasta los 90 días después de aplicado los tratamientos, presenta un crecimiento ascendente en función del tiempo de evaluación (Figura 2) presentando un índice de correlación $\mathrm{R}^{2} \mathrm{de}$ 0.979; determinando que existe un grado de asociación importante y positiva entre días de evaluación y longitud de brotes (cm); resultado esperado y de respuesta normal en trabajo de podas de formación.

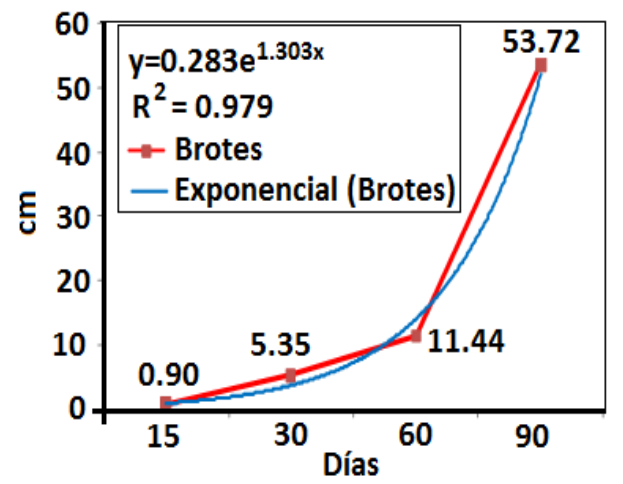

Figura 2. Curva de regresión exponencial para la variable longitud $(\mathrm{cm})$ de brotes.
En la Figura 3 se observa la respuesta de las plantas de camu camu después de 90 días de haber aplicado el primer corte de poda a diferentes alturas del tallo. Efectivamente el testigo sin poda es el que tuvo mayor $\mathrm{N}^{\mathrm{o}}$ de brotes pero de menor longitud.

Por otra parte, las plantas que fueron podados a 10, 20 y $40 \mathrm{~cm}$ de altura poseen menor cantidad de brotes, pero de mayor longitud el cual permitirá realizar la formación de la estructura o esqueleto para conseguir árboles bien formados $\mathrm{y}$ equilibrados para obtener la máxima producción con la mejor calidad de frutos posible (Mataix y Villarrubia, 1999).

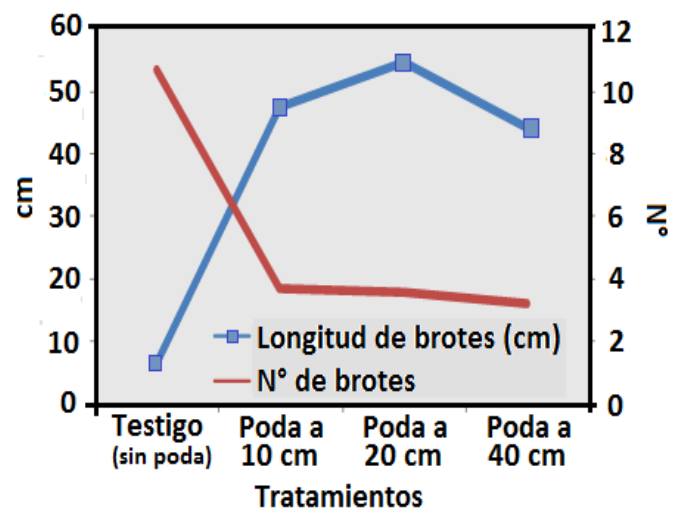

Figura 3. Respuesta inicial de las plantas de camu camu a la primera poda de formación a diferentes alturas.

Luego se realizó el raleo de brotes dejando en promedio 3 a 4 por planta. Fueron podados a $30 \mathrm{~cm}$ (Barrantes, 2006) y cada tres meses escalonadamente (López y Casanova, 2006).

Después de haber realizado la cuarta poda de formación y su correspondiente evaluación, el tratamiento que alcanzó la mayor altura $(\mathrm{cm})$ y diferencias significativas con los demás tratamientos, a un nivel de confianza superior al $95 \%$, fue el Testigo sin poda. Esto puede deberse a que estas plantas fueron instaladas con una altura inicial (ai) de $154 \mathrm{~cm}$ y además no fueron podadas. 


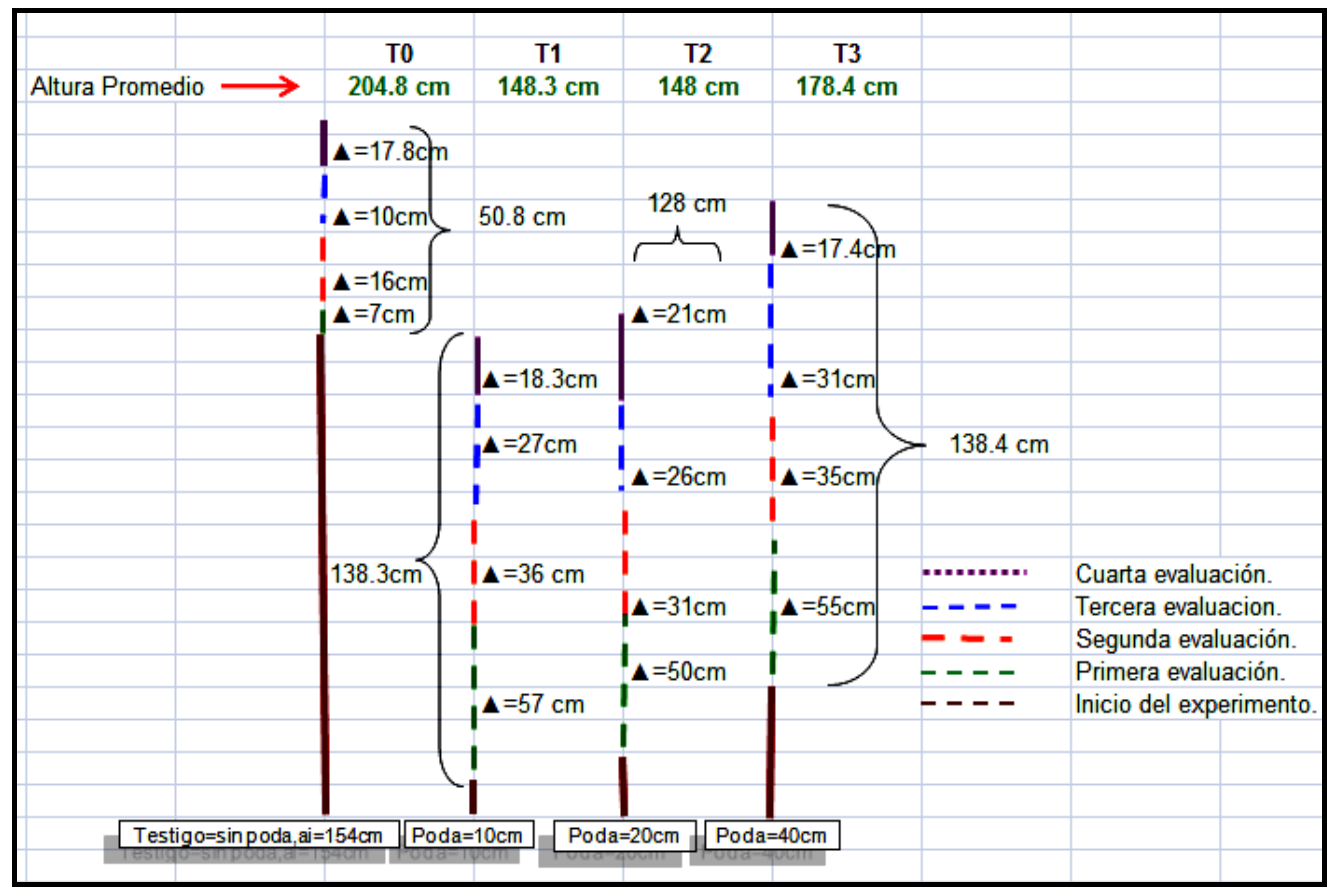

Figura 4. Incremento de altura $(\mathrm{cm})$ de las plantas de camu camu por efecto de las podas de formación.

Entre los tratamientos con poda, el T3 destaca por presentar inicialmente un nivel de poda mayor, no obstante en relación al incremento de altura $(\mathrm{cm})$, se comportaron de forma similar, superando al Testigo sin poda en $267 \%$ por presentar sólo un crecimiento de $50.8 \mathrm{~cm}$. Las plantas de los tratamientos con poda tuvieron un crecimiento por encima de $128 \mathrm{~cm}$. Se puede afirmar que la poda de formación incrementa considerablemente la altura de planta (Figura 4). En el comportamiento del diámetro basal (Figura 5), se observa que el tratamiento con Poda a $10 \mathrm{~cm}$ logró superioridad con $1.96 \mathrm{~cm}$, presentando tres niveles de significancia menor al $0.05 \%$ con los tratamientos Sin poda, T2 y T3 (Tabla 8).

Estos resultados son bastante ajustados a los reportes de la literatura (López y Casanova, 2006), donde se argumenta que mientras el corte sea a menor longitud de ramas la respuesta se orienta al engrosamiento rápido del tallo y a la emisión de brotes. También estos resultados nos pueden ayudar a suponer que a medida que la longitud de corte sea menor desde la base del suelo el diámetro basal será mayor.

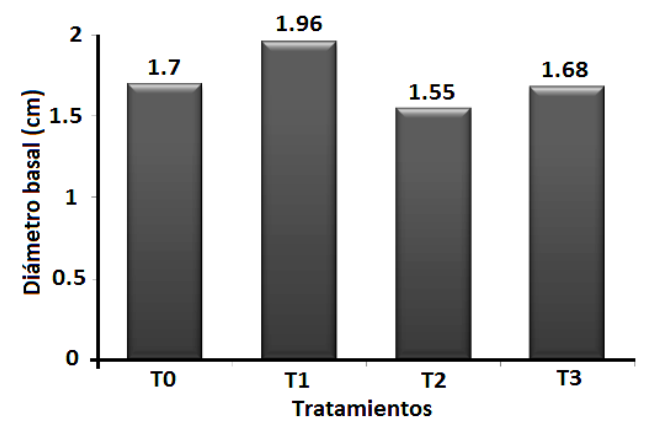

Figura 5. Diámetro Basal promedio $(\mathrm{cm})$ por tratamiento.

\section{Tabla 8}

Prueba de Tukey para el diámetro basal por efecto del tratamiento.

\begin{tabular}{lcccc}
\hline \multirow{2}{*}{ DHS de Tukey a,b,c } & & \multicolumn{3}{c}{$\begin{array}{c}\text { Significación } \\
(\alpha=0.05)\end{array}$} \\
\cline { 2 - 5 } & & \multicolumn{2}{c}{ Subconjunto $-15 \mathrm{~d}$} \\
\hline Tratamiento & Promedio & 1 & 2 & 3 \\
\hline T0 [Testigo sin poda] & 56 & $\mathrm{a}$ & & \\
T1 [Poda a $10 \mathrm{~cm}$ ] & 58 & & $\mathrm{~b}$ & \\
T2 [Poda a $20 \mathrm{~cm}$ ] & 57 & & $\mathrm{~b}$ & \\
T3 [Poda a $40 \mathrm{~cm}$ ] & 58 & & & $\mathrm{c}$ \\
\hline
\end{tabular}


Tabla 9

Análisis de Varianza para el diámetro de copa (cm) de plantas de camu camu.

\begin{tabular}{lcccc}
\hline Variable & Fuente & GL & F & Significación \\
\hline \multirow{3}{*}{ Diámetro de } & Tmto. & 3 & 2.865 & 0.038 \\
copa $(\mathrm{cm})$ & Rep. & 2 & 1.063 & 0.347 \\
& Error & 214 & & \\
& Total & 219 & & \\
\hline
\end{tabular}

El diámetro de copa de las plantas Testigo desatacaron con $90.91 \mathrm{~cm}$ en promedio (Figura 6). Probablemente se debió a que las ramas se elongaron más por el peso y por la poca consistencia que tienen (Figura 7), el resto de tratamientos tienen valores similares comprendidos entre 78 y $79.5 \mathrm{~cm}$.

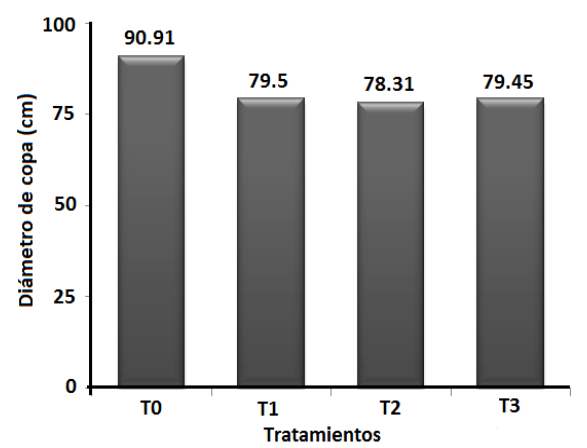

Figura 6. Diámetro de copa promedio $(\mathrm{cm})$ por tratamiento.

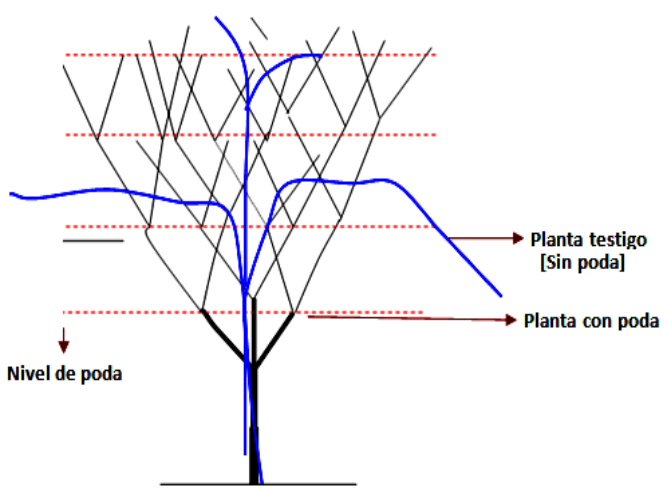

Figura 7. Superposición entre una planta con poda y otra sin poda para diferenciar el dímetro de copa.

En el análisis de variancia para la variable Número de ramas totales (evaluados al año 2010) se observa que existen diferencias significativas entre tratamientos a un nivel de confianza superiores al 95\% (Tabla 10).

\section{Tabla 10}

Análisis de Varianza para el número de ramas por efecto del tratamiento en plantas juveniles de camu camu.

\begin{tabular}{lcccc}
\hline Variable & Fuente & GL & F & Significación \\
\hline \multirow{3}{*}{$\mathrm{N}^{\circ}$ ramas } & Tmto. & 3 & 23.48 & 0.008 \\
& Rep. & 2 & 191.87 & 0.90 \\
& Error & 213 & & \\
& Total & 218 & & \\
\hline
\end{tabular}

En la Tabla 11 se observa que el mayor número de ramas totales lo obtuvieron los tratamientos T1 [Poda a $10 \mathrm{~cm}$ ], T3 [Poda a $40 \mathrm{~cm}$ ] y T2 [Poda a $20 \mathrm{~cm}$ ], con 13 y 14 unidades siendo significativamente superiores que T0 [sin poda], que solo alcanzó un valor promedio de 3 ramas debido a que no se le practicó ningún tipo de poda. Con estos resultados se puede afirmar que la poda logra incrementar en más de $432.3 \%$ el número de ramas frente al T0 [Testigo sin poda] (Figura 8, Figura 9 y Figura 10), con lo cual se podrá formar adecuadamente la estructura o esqueleto para soportar la capacidad productiva (Mataix y Villarrubia, 1999).

\section{Tabla 11}

Prueba de Tukey para el número de ramas por efecto del tratamiento.

\begin{tabular}{lccc}
\hline \multirow{2}{*}{ DHS de Tukey ${ }^{\mathrm{a}, \mathrm{b}, \mathrm{c}}$} & & \multicolumn{2}{c}{$\begin{array}{c}\text { Significación } \\
(\alpha=0.05)\end{array}$} \\
\cline { 2 - 4 } & & \multicolumn{2}{c}{ Subconjunto } \\
\hline Tratamiento & Promedio & 1 & 2 \\
\hline T0 [Testigo sin poda] & 3 & $\mathrm{a}$ & \\
T1 [Poda a $10 \mathrm{~cm}$ ] & 14 & & $\mathrm{~b}$ \\
T2 [Poda a $20 \mathrm{~cm}$ ] & 13 & & $\mathrm{~b}$ \\
T3 [Poda a $40 \mathrm{~cm}$ ] & 14 & & $\mathrm{~b}$ \\
\hline
\end{tabular}

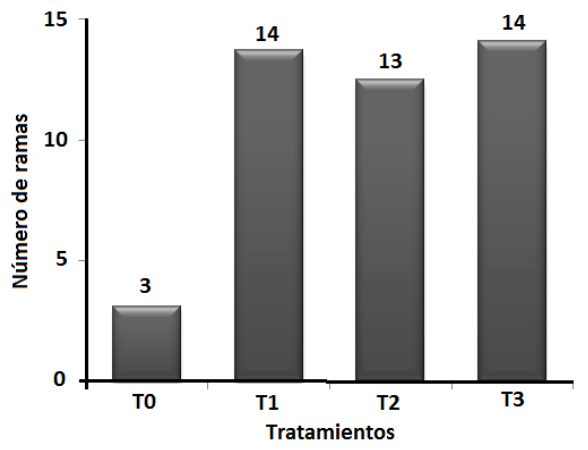

Figura 8. Número de ramas promedio por tratamiento. 


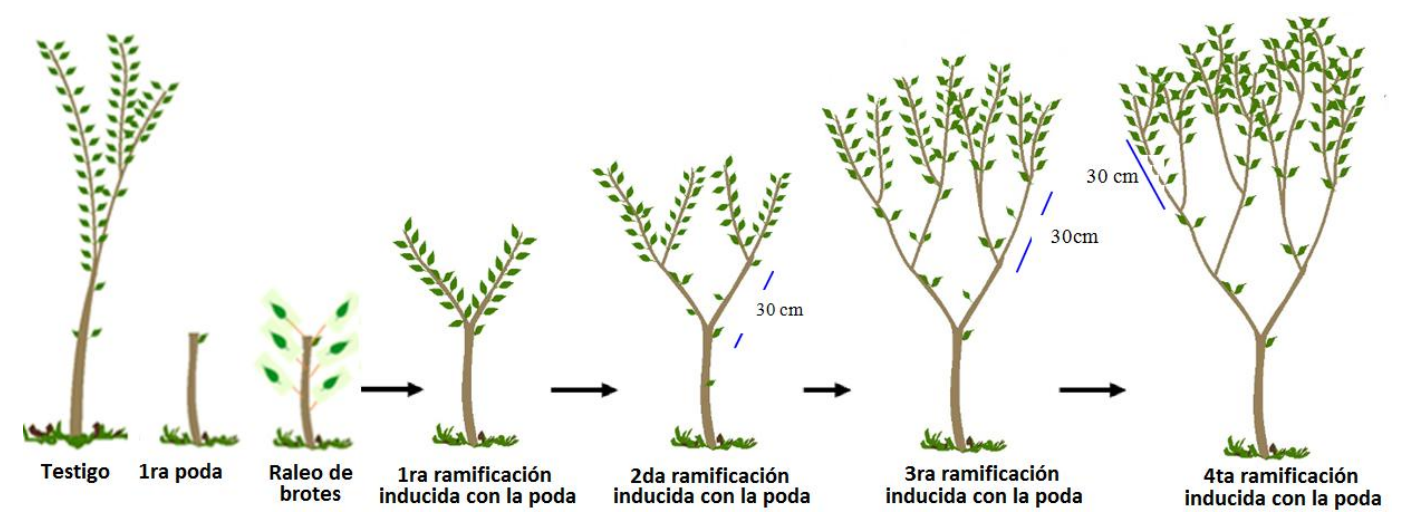

Figura 9. Podas de formación arquitectónica en plantas de camu camu.
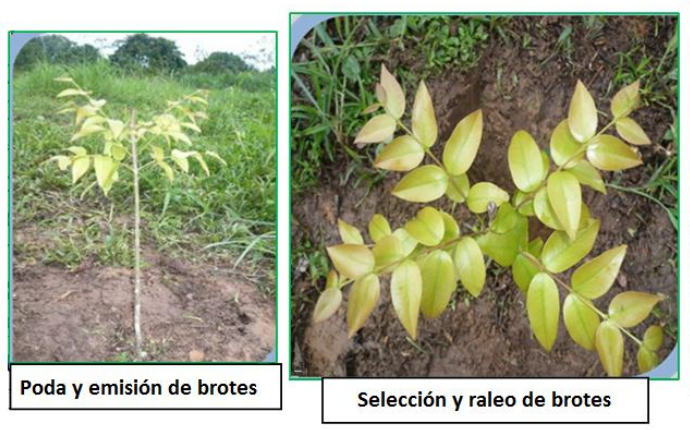

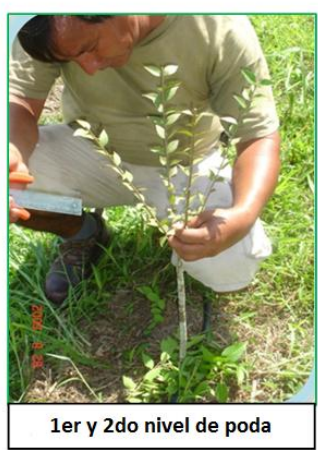

1er y 2 do nivel de poda

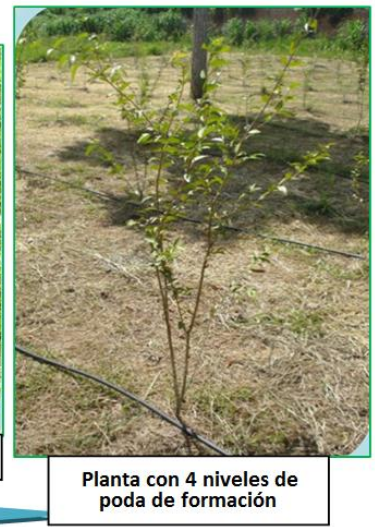

Figura 10. Pasos para formar la copa en plantaciones iniciales de camu camu.

\section{Conclusiones}

La mayor cantidad de brotes lo obtuvieron las plantas testigo sin poda; sin embargo las plantas con poda destacaron por presentar mayor longitud de brotes. Se encuentra mayor crecimiento de brotes cuando las plantas son podadas a $20 \mathrm{~cm}$ de altura desde la base del tallo. Se logró conseguir un incremento de altura $(\mathrm{cm})$ en las plantas con poda; en más de $267 \%$ frente al tratamiento Testigo sin poda. Las diferencias entre altura de la planta y diámetro basal después de la poda de formación son atribuidas a los efectos del tratamiento. El diámetro basal es mayor a medida que el primer nivel de poda sea a menor altura desde la base del suelo. El tratamiento testigo sin poda presentó mayor diámetro de copa atribuida a la elongación de las ramas, peso y la poca estabilidad. Con la poda de formación se incrementó considerablemente el número de ramas en más de $432.3 \%$ frente al tratamiento testigo sin poda.

\section{Referencias}

Abanto, C. 2011. Efecto del fertirriego sobre la productividad del camu camu (Myrciaria dubia H.B.K Mc Vaugh) en la Región de Ucayali. Tesis (Ing. Forestal) Pucallpa PE: Universidad Nacional Agraria la Molina-Instituto de Investigaciones de la Amazonia Peruana, 96p.

Abanto, C. 2010. Informe técnico final: Estudio del efecto de las podas de formación arquitectónica en plantaciones iniciales de camu camu (Myrciaria dubia H.B.K. Mc Vaugh), en la Región de Ucayali - Perú, $26 \mathrm{p}$.

Avilán, L.; Rodríguez, M.; Ruiz, J. 2000. El mango se poda: ¿por qué, cuándo y cómo? FONAIAP Divulga $\mathrm{N}^{\circ}$ 65. Consultado 1 de Enero del 2011.Disponible en: http://sian.inia.gob.ve/repositorio/revistas_tec/Fonaiap Divulga/fd65/texto/mango.htm 
Barrantes, N. 2006. Poda y ajuste de la época de producción en el cultivo de Guayaba. Ministerio de Agricultura y Ganadería. Región pacifico central. Agencia de servicios agropecuarios de Jicaral. 4 pp. Consultado 1 de Enero del 2011.Disponible en: http://www.mag.go.cr/bibioteca_virtual_ciencia/paccentr-bol-guayaba.pdf

IIAP. 2001. Sistema de Producción de Camu camu en Restinga. Programa de Ecosistemas terrestres. Proyecto Bioexport. Camu camu. 60 pág.

Mataix, E; Villarrubia, D. 1999. Poda de Frutales. Serie de divulgación técnica $\mathrm{N}^{\circ}$ 45, I.S.B.N.: 84-482-2291-1, Depósito Legal: V-4154-1999. Consejería de Agricultura, Pesca y Alimentación-Dirección General de Innovación Agraria y Ganadería, 104 pág. (en línea). Consultado 12 Julio del 2011. Disponible en: http://www.ivia.es/sdta/pdf/libros/n45.pdf

Morris, D.; Suárez, C. S.f. Plantación y poda de frutales. Estación Experimental Agropecuaria Bordenave Unidad de Comunicaciones Bahía Blanca 1 pág. (en línea). Disponible en: http://www.inta.gov.ar/bordenave/info/indices/ tematica/extension/plantacion_poda_de_frutales.pdf.
López, D.; Casanova, E. 2006. Manual Poda y sistemas de formación en los frutales de hueso Centro Integrado de Formación y Experiencias Agrarias de Jumilla, 28 pág. (en línea). Consultado 12 Julio del 2011. Disponible en: http://www.ruralnaturaleza.com/ files/frutales_de_hueso.pdf

PROMPEX. 1998. El cultivo del camu camu en la Amazonía peruana. Promoción de Exportaciones de Productos Agrícolas de la Selva. Citado por el proyecto Asesoría en Planeación Agraria. (PROAPAGTZ). Estudio de Mercado para Myrciaria dubia H.B.K. Mc Vaugh (camu camu), 2006. 46 pág.

Zeledon, R.; Wan, F.J. 1994. El cultivo de la guayaba. Cañas Guanacaste, Costa Rica. 3 pág. Consultado 1 de Enero del 2011. Disponible en: http://www.mag.go.cr/bibioteca_virtual_ciencia/tec_gu ayaba.pdf 